\title{
Trauma workload during COVID19 lockdown: an analysis of incidence in 4 million people
}

\author{
Alessandro Aprato ${ }^{1}$ (]) $\cdot$ Nathalie Bini ${ }^{1} \cdot$ Silvia Ferro $^{2} \cdot$ Lucia Favella $^{2} \cdot$ Luigi Conforti $^{1} \cdot$ Alessandro Massè $^{1}$
}

Received: 13 November 2020 / Accepted: 7 February 2021 / Published online: 17 February 2021

(c) Royal Academy of Medicine in Ireland 2021, corrected publication 2021

\begin{abstract}
Background Aim of this study is to report the trauma workload during COVID19 lockdown in a region of four million people and to compare it with the same period in 2019.

Methods The regional register for A\&E admissions and hospitalizations has been reviewed in order to compare the number of A\&D admission, the triage colour codes rates, aetiology of trauma, number of patients hospitalized for trauma, number of fractures that required surgery, type of fractures and injuries and mean patients' age.

Results During lockdown 7314 patients were admitted in A\&E, while 22,508 patients were admitted in 2019. In 2020 and 2019 triage codes were respectively distributed as follows: red code $0.1 \%$ vs $0.2 \%$, yellow code $8.9 \%$ vs $6.3 \%$, green code $84 \%$ vs $84.7 \%$ and white code $6 \%$ vs $8.8 \%$. $(p=0.042)$. The number of hospitalized patients for trauma was 670 in 2020 , while in 2019 was $1774(p=0.02)$. The most common fracture that required surgery was femur fracture (409 in 2020 vs 635 in 2019); fracture subtype distribution and mean age of the patients were significantly different in the two groups (respectively $p<0.01$ and $p=0.02$ ).

Conclusions One month of lockdown showed a $68 \%$ decrease in the number of A\&E visits and a $74 \%$ decrease of fractures that required surgery. Femur fracture showed the lowest decrease moving from 635 to 409 units but increasing their incidence rate $(42$ to $61 \%)$.
\end{abstract}

Keywords COVID19 $\cdot$ Fracture $\cdot$ Injury $\cdot$ Lockdown $\cdot$ Trauma

\section{Background}

Trauma epidemiology is expected to change radically during a lockdown, as showed in Wong et al. recent study [1]. We set out this study to examine the implications of COVID19 lockdown in trauma patients in an Italian region of four million people (Piedmont). After the spread out of the COVID19 virus, in Italy the first lockdowns began around 21 February 2020 [2], covering a single province in Lombardy; later on the 8 March 2020 [3], Italian prime minister announced the expansion of the quarantine zone to cover much of northern Italy including the full Piedmont region. The lockdown restricted travel from, to or within the affected areas, banned funerals and cultural events, and requiring

\footnotetext{
Alessandro Aprato

ale_aprato@hotmail.com

School of Medicine, University of Turin, Turin, Italy

2 Piedmont Regional Health Center, Turin, Italy
}

people to keep at least $1 \mathrm{~m}$ of distance from one another in public locations. People would still be able to use trains and planes to and from the region only for "proven work needs, emergencies, or health reasons." On 22 March, Italian prime minister announced a further enlargement of the lockdown, by shutting down all non-necessary businesses and industries [4]. The aim of this study is to report the trauma workload during COVID19 lockdown in a region of four million people and to compare it with the same period in 2019.

\section{Methods}

This retrospective study was performed by the cooperation between the regional I level trauma centre and the Regional Health Centre. The study protocol was approved by the local committee and by The National Committees for Research Ethics in Italy (2018/20121), trial number 287.718 (14/04/2020). 
The regional register for $\mathrm{A} \& \mathrm{E}$ visits and hospitalization collects anonymized data and is held by the Regional Health Centre as standard procedure for health government; revision of the elaborated data was performed by regional I level trauma centre. Research staff working in the analysis of the results was not involved in data collection. All patients admitted to one of the regional A\&E Departments are registered in the regional register for A\&E visits and hospitalizations. This register is collected from the regional government for economic and political reasons since 2013 [5]. The register collects the following data: age, sex, date of A\&E admission, A\&E Department, colour code at initial triage (white, green, yellow, red), diagnoses, diagnoses code (ICD9CM), discharge date and type of discharge (hospitalization or discharged at home).

Criteria of inclusion in this study were admission date from 8 March 2020 to 7 April 2020 for study group and admission date from 8 March 2019 to 7 April 2019 for comparison group; diagnosis code (ICD9CMInternational Classification of Diseases 9th revision Clinical Modification) [6] included in the "injury and poisoning" chapter that covers ICD codes 800 to 999 (pages 473 to 546 of Volume 1).

Records were excluded if the only diagnosis code (ICD9CM) was one of the following lists:

- Fracture of skull (code between 800 and 804)

- Intracranial injury, excluding those with skull fracture (code between 850 and 854)

- Internal injury of thorax, abdomen, and pelvis (code between 860 and 869$)$

- Injury to blood vessels (code between 900 and 904)

- Late effects of injuries, poisonings, toxic effects, and other external causes (code between 905 and 909)

- Effects of foreign body entering through Body orifice (code between 930 and 939)

- Burns (code between 940 and 949)

- Poisoning by drugs, medicinal and biological substances (code between 960 and 979)

- Toxic effects of substances chiefly non-medicinal as to source (code between 980 and 989

- Other and unspecified effects of external causes (code between 990 and 995)

- Complications of surgical and medical care, not elsewhere classified (code between 996 and 999)

Patients were dived into two groups: 2019 and 2020. Groups were compared in term of age, sex, number of A\&E visits, triage codes, number of hospitalization, number of fractures that required surgery and distribution of fractures (and subtypes) that required surgery, causes of fracture, age of femur fracture and type of discharge.
Categorical data were reported as frequencies and percentage, while continuous variables as mean and standard deviation when the distribution was normal or median and IQR if the distribution was not normal. The normality of data was verified with the Shapiro-Wilk test. Two group comparisons were performed with the chi squared test for categorical data. For continuous data Student $t$ test or Mann-Whitney test were used according to the distribution of the values. The statistical analysis was performed using StataMP13 (Stata Corp., College Station, TX.)

\section{Results}

During the first month of lockdown (from 8 March 2020 to 7 April 2020), 7314 patients were admitted into one of the regional A\&E Departments, while in the same period in 2019, 22,508 patients were admitted. Daily distribution of the A\&E visits shows a significant drop of admission after 4 days from Lockdown (Fig. 1).

Mean ages of the two groups were significantly different: 73.2 years (SD 19.3) in group 2020 vs 63.5 years (SD $22.7)$ in group $2019(p=0.02)$. Sex distribution was not significantly different in the two groups (58\% of female vs $56 \%, p=0.75$ ).

In 2020 triage codes were $0.1 \%$ red code, yellow code $8.9 \%$, green code $84 \%$ and white code $6 \%$. In 2019 distribution was as follows: red code $0.2 \%$, yellow code $6.3 \%$, green code $84.7 \%$ and white code $8.8 \%$. Distribution was significantly different between the two periods $(p=0.042)$.

The number of hospitalized patients for trauma was 670 in the study period (2020), while in the same period of 2019 was $1774(p=0.02)$. Distributions of hospitalization rates were significantly different ( $p=0.02$ ): in $20209.2 \%$ were hospitalized and $89.6 \%$ were discharged at home, while in $20194.4 \%$ of patient were hospitalized and $94.8 \%$ were discharged at home.

Numbers and distribution of fractures/injuries was significantly different in the two groups: neck distractions

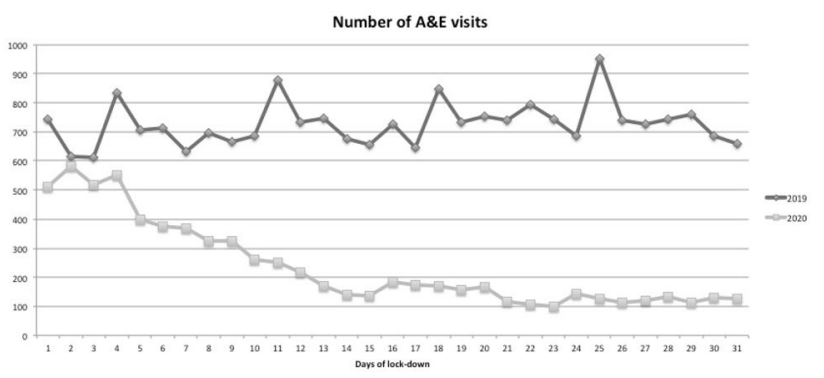

Fig. 1 Daily distribution of the A\&E visits in the same period of 2019 and 2020 
Table 1 Top 10 causes of A\&E orthopaedic visits in 2019 and 2020

\begin{tabular}{cll}
\hline Ranking & Diagnosis & Frequency (Percentage) \\
\hline 2019 & & \\
1 & Neck distraction & $2560(6.36 \%)$ \\
2 & Ankle distraction & $1758(4.34 \%)$ \\
3 & Finger wound & $1380(3.40 \%)$ \\
4 & Contusion (site not specified) & $1276(3.15 \%)$ \\
5 & Knee contusion & $1258(3.10 \%)$ \\
6 & Finger contusion & $1083(2.67 \%)$ \\
7 & Wrist contusion & $842(2.08 \%)$ \\
8 & Finger distraction & $830(2.05 \%)$ \\
9 & Shoulder contusion & $778(1.91 \%)$ \\
10 & Hand contusion & $774(1.91 \%)$ \\
2020 & & \\
1 & Finger wound & $373(5.1 \%)$ \\
2 & Neck distraction & $254(3.47 \%)$ \\
3 & Proximal femur fracture & $208(2.84 \%)$ \\
4 & Ankle distraction & $195(2.67 \%)$ \\
5 & Contusion (not specified) & $191(2.61 \%)$ \\
6 & Knee contusion & $166(2.27 \%)$ \\
7 & Complex finger wound & $152(2.08 \%)$ \\
8 & Multiple contusions & $130(1.78 \%)$ \\
9 & Hand contusion & $119(1.63 \%)$ \\
10 & Wrist contusion & $113(1.54 \%)$ \\
\hline
\end{tabular}

were reduced by $90 \%$, ankle distractions by $89 \%$, knee contusions by $90 \%$ and wrist contusions by $90 \%$ (Table 1 ). Finger wounds were the injury with the lowest number reduction $(-73 \%)$.

Comparison of specific fractures/injuries that required surgery showed a significant drop of humerus fractures $(-72 \%)$, forearm fractures $(-85 \%)$, tibias $(-66 \%)$, ankles $(-88 \%)$, acromion-clavicular dislocations $(-98 \%)$ and anterior cruciate ligament injury $(-97 \%)$, while femure fracture numbers reduced only by $36 \%$ (Table 2 ).

Table 2 Frequency and percentage of specific fractures/injuries that required surgery

\begin{tabular}{llllll}
\hline & \multicolumn{2}{l}{ Frequency } & & \multicolumn{2}{l}{ Percentage } \\
\cline { 2 - 3 } \cline { 6 - 7 } \cline { 6 - 7 } & 2019 & 2020 & & 2019 & 2020 \\
\hline Humerus fracture & 92 & 26 & & 6.08 & 3.88 \\
Forearm fracture & 137 & 20 & & 9.05 & 2.99 \\
Femur fracture & 635 & 409 & & 41.94 & 61.05 \\
Tibia fracture & 125 & 43 & & 8.26 & 6.42 \\
Ankle fracture & 119 & 14 & & 7.86 & 2.09 \\
Acromion-clavicular dislocation & 54 & 1 & & 3.57 & 0.15 \\
Anterior cruciate ligament injury & 69 & 2 & & 4.56 & 0.30 \\
Other & 543 & 155 & & 18.68 & 23.12 \\
Total & 1774 & 670 & & 100 & 100 \\
\hline
\end{tabular}

Among femur fractures, subtype distribution was significantly different in 2020 vs 2019: medial, lateral and subtrochanteric fractures were respectively $35 \%, 43 \%$ and $22 \%$ in 2020, while in 2019 they were respectively $46 \%$, $45 \%$ and $9 \%(p<0.01)$. Mean age in femur fracture patient was 87.3 year (SD 10.2) in 2020 and 83.6 years (SD 12.2) in $2019(p=0.02)$.

Main causes of fracture in 2020 were 55\% falls at home, not specified (other causes) in $0.04 \%$ and $1 \%$ intentional trauma, while in 2019 those rates were respectively were $8 \%, 65 \%$ and $0.01 \%$.

\section{Conclusions}

The public health impact of our findings is significant. The number of A\&E visits for orthopaedic showed a significant decrease $(68 \%)$ comparing traumas in the first month of lockdown (2020) with the same period in 2019. This result is in line with the findings found in a study developed in 15 Italian first- and second-level trauma centres where a decrease of $71 \%$ of A\&E visits was observed [7] but also in non-traumatic patients: Thornton [8] reported an overall $25 \%$ decrease of A\&E visits in England in the first week after lockdown independently from the cause of admissions (excluding COVID19-related causes). Those figures confirm that the public is frightened of going to emergency departments and the Royal College of Emergency Medicine already underlined that some people may be harmed by not accessing treatment [9].

Causes of A\&E visits were different in the two groups: neck distraction, ankle distraction and knee contusion that were between the most common diagnoses in 2019 reduced their incidence rates (respectively $-45 \%,-38 \%$ and $-26 \%$ ). These reductions are related to decreasing in road traffic (55\% less on Italian highways on March 2020 [10]), outdoor activities and workplace injuries. On the other hand, the incidence rate of finger wounds increased significantly $(+50 \%)$ that may be due to the increase of the home hobbies during the lockdown: food processing equipment, lawn mower and power saw (activities usually practiced at home) are reported among the top 5 mechanisms in those aged over 15 years in non-lockdown period [11]).

Our results showed also a decrease of $74 \%$ in the overall number of fractures that required surgery. The decreased road traffic and outdoor activities may play a central role in the interpretation of this finding. Although not unexpected, we must underline that femur fracture showed a decrease in the absolute numbers (moving from 635 to 409 units), but it was the only fracture that increased its distribution rate among all the surgical fractures (from 42 to $61 \%$ ). This may justify the significant increase of mean patients age between 2019 and 2020 (73.2 vs 83.5 years $p=0.02$ ). According to 
our opinion and as showed in literature [12], age is directly related to fracture mechanism: fractures due to bone fragility rather than severe injury are uncommon in young adults [13]. Locking-down reduced the mobility of mild-elderly patients who are not getting outside their home so much reducing the incidence of their femur fractures. Instead, older patients fall at home as before COVID19 pandemic. Furthermore, among femur fractures, subtrochanteric fractures significantly increased their incidence (9\% in 2019 vs $22 \%$ in 2020 , $p<0.01$ ). This finding agrees with the highest incidence of those fractures in fragile patients: Peritrochanteric femur fractures typically occur in a physiologically older population than femoral neck fractures [14].

According to our point of view, femur fractures during lockdown were restricted to fragile patients because the more active patients at risk of falling during outdoor daily activities reduced their movements during the lockdown and occurred less frequently in femur fracture. This theory also justify that mean age in femur fracture patient was 83.6 years (SD 12.2) in 2019 and 87.3 year (10.2) in 2020 $(p=0.02)$. Further studies should focus on the American Society of Anesthesiologists-ASA score to confirm or deny our hypothesis: femoral fractures during lockdown are restricted to more fragile patients. This hypothesis is in line with Gumina et al. study [15] where elderly subjects remain exposed to shoulder and elbow trauma due to low-energy (domestic) falls.

A\&E accesses shift from a 6.3 to an $8.9 \%$ of yellow codes, while white codes decreased from 8.8 to $6 \%$; the percentage of green codes were similar $(84.7 \%$ vs $84.0 \%)$. This finding suggests that $A \& E$ admission in this region were in part inappropriate as already described in previously published paper [16].

According to our point of view, the fear of COVID19 transmission inside the hospitals limited the A\&E consultation to the most urgent ones.

Major sporting events can simulate somehow a situation of lockdown during which people go out only for urgent reasons and several studies demonstrated a decrease in emergency department attendance for non-urgent cases [17-21].

Eventually, a huge amount of mental health studies has been carried out empathizing the need of a pre-organised health care system [22]. People with mental health conditions could be more substantially influenced by the emotional responses brought on by the COVID19 epidemic, resulting in relapses or worsening of an already existing mental health condition also due to the quarantine measures affecting a regular follow up [23]. Moreover, stress, anxiety, and stigmatization were commonly reported in infected individuals and health care workers during Sars [24]. We can assume that this is also happening now during COVID19 pandemic, resulting in an increased number of people at risk for impulsive actions.
We think our study results must be taken into consideration when re-organizing trauma during a lockdown period.

Funding Financial support for this study was obtained from University of Turin.

\section{Declarations}

Ethical approval The study protocol was approved by the local committee and by the National Committees for Research Ethics in Italy (2018/20121), trial number 287.718 (14/04/2020). All procedures performed in studies involving human participants were in accordance with the ethical standards of the institutional and/or national research committee and with the 1964 Helsinki declaration and its later amendments or comparable ethical standards.

Conflict of interest The authors declare that they have no conflict of interest.

\section{References}

1. Wong JSH, Cheung KMC (2020) Impact of COVID-19 on orthopaedic and trauma service: an epidemiological study. J Bone Joint Surg Am 102(14):e80. https://doi.org/10.2106/ JBJS.20.00775.PMID:32675668;PMCID:PMC7431143

2. Ministero della Salute. (20A01220). Ulteriori misure profilattiche contro la diffusione della malattia infettiva COVID-19. https:// www.gazzettaufficiale.it/eli/id/2020/02/22/20A01220/sg

3. Decreto del presidente del consiglio dei ministri. (20A01522) Ulteriori disposizioni attuative del decreto-legge 23 febbraio 2020 , n. 6, recante misure urgenti in materia di contenimento e gestione dell'emergenza epidemiologica da COVID-19. https:// www.gazzettaufficiale.it/eli/id/2020/03/08/20A01522/sg

4. Decreto del presidente del consiglio dei ministri. Ulteriori disposizioni. http://www.governo.it/sites/new.governo.it/files/ dpcm_20200322.pdf

5. Deliberazione della Giunta Regionale (2013) Organizzazione delle attività di controllo analitico delle cartelle cliniche e delle schede di dimissione ospedaliera ai sensi dell' art. 79, comma 1 septies del D.L. 112 del 2008 convertito con modificazioni, dalla legge n 133 del 6.8.2008 e conseguenti modalità di revisione tariffaria. Modificazioni delle DD.G.R. 5.9.2011 n. 35-6651, n. 19-2561, e .1.2.2010, n. 7-13150. http://www.regione.piemonte.it/governo/ bollettino/abbonati/2013/49/attach/dgr_06651_830_11112013.pdf

6. Ministero della salute. Manuale ICD9-CM. http://www.salute. gov.it/portale/temi/p2_6.jsp?lingua=italiano\&id=1278\&area= ricoveriOspedalieri\&menu=classificazione

7. Benazzo F, Rossi SMP, Maniscalco P et al (2020) The orthopaedic and traumatology scenario during Covid-19 outbreak in Italy: chronicles of a silent war. Int Orthop. 2020 Aug;44(8):1453-1459. doi: https://doi.org/10.1007/s00264-020-04637-3. Epub 2020 Jun 26. PMID: 32591960; PMCID: PMC7319215

8. Thornton J (2020) Covid-19: A\&E visits in England fall by $25 \%$ in week after lockdown. BMJ 369:m1401. https://doi.org/10.1136/ bmj.m1401

9. Royal College of Emergency Medicine (2020) Seriously ill or injured patients may be avoiding Emergency Departments due to Covid-19 fears". https://www.rcem.ac.uk/RCEM/News/ News_2020/RCEM__seriously_ill_or_injured_patients_may_ be_avoiding_Emergency_Departments_due_to_Covid-19_fears. aspx 
10. Il Messaggero (2020) Coronavirus, Anas: a marzo calo traffico del 55\%. Per i mezzi pesanti meno 25\%. https://www.ilmessaggero.it/ economia/news/coronavirus_anas_marzo_calo_traffico_ mezzi_pesanti_contenuto-5158039.html

11. Reid DBC, Shah KN, Eltorai AEM, Got CC, Daniels AH (2019) Epidemiology of finger amputations in the United States from 1997 to 2016. Journal of Hand Surgery Global Online, 1(2). ISSN 45-51:2589-5141. https://doi.org/10.1016/j.jhsg.2019.02.001

12. Wu S, Rau C, Kuo SCH (2019) The influence of ageing on the incidence and site of trauma femoral fractures: a cross-sectional analysis. BMC MusculoskeletDisord 20:413. https://doi. org/10.1186/s12891-019-2803-x

13. Office of the Surgeon General (US) (2004) Bone health and osteoporosis: a report of the surgeon general. Rockville (MD): Office of the Surgeon General (US); 2, The Basics of Bone in Health and Disease. https://www.ncbi.nlm.nih.gov/books/NBK45504/

14. Babcock S, Kellam JF (2018) Hip fracture nonunions: diagnosis, treatment, and special considerations in elderly patients. Advances in orthopedics, 1912762. https://doi. org/10.1155/2018/1912762

15. Gumina S, Proietti R, Polizzotti G et al (2020) The impact of COVID-19 on shoulder and elbow trauma: an Italian survey. J Shoulder Elbow Surg. 29(9):1737-1742. https://doi.org/10.1016/j. jse.2020.05.003. Epub 2020 May 20. PMID: 32713663; PMCID: PMC7237917

16. Fornero G, Arione R, Cibinel GA, Ripa F (2015) Accessi inappropriati e sovraffollamento del pronto soccorso: uno studio multicentrico nella Regione Piemonte, $10^{\circ}$ Conferenza GIMBE, Aumentare il value e ridurre gli sprechi in sanità.
17. Reich NT, Moscati R, Jehle D, Ciotoli M (1994) The impact of a major televised sporting event on emergency department census. J Emerg Med 12:15-17

18. Jerrard DA (2009) Male patient visits to the emergency department decline during the play of major sporting events. West J Emerg Med 10:101-103

19. Redelmeier DA, Vermeulen MJ (2011) Emergency department visits during an Olympic gold medal television broadcast. Open Med 5:e112-e119

20. Reis BY, Brownstein JS, Mandl KD (2005) Running outside the baseline: impact of the 2004 Major League Baseball postseason on emergency department use. Ann Emerg Med 46:386-387

21. Savry C, Oger E, Chahir N (2001) Effect of the 1998 Soccer World Cup tournament on rescue requests. Intensive Care Med 27:1224-1226

22. Douglas PK, Douglas DB, Harrigan DC, Douglas KM (2009) Preparing for pandemic influenza and its aftermath: mental health issues considered. Int J Emerg Ment Health 11(3):137-44

23. Hao Y, Jian-Hua C, Yi-Feng X (2020) Patients with mental health disorders in the COVID-19 epidemic. https://www.thelancet.com/ psychiatryhttps://doi.org/10.1016/S2215-0366(20)30090-0

24. Maunder R, Hunter J, Vincent L et al (2003) The immediate psychological and occupational impact of the 2003 SARS outbreak in a teaching hospital. CMAJ 168(10):1245-1251 